Поступила в редакцию 05.04.2021

Подписана в печать 20.11.2021

\title{
МНОГОКАНАЛЬНАЯ МОДЕЛЬ ПРОЦЕССА ТЕПЛОПРОВОДНОСТИ
}

\author{
(C) 2021 А. С. Степашкина
}

\section{Санкт-Петербургский государственный университет аэрокосмического приборостроения ул. Большая Морская, 67, лит. А, 190000 Санкт-Петербург, Российская Федерация}

\begin{abstract}
Аннотация. Описание процесса теплопереноса на макроскопическом уровне производится с помощью хорошо известных классических методов и теорий, найденных либо путем аппроксимации опытных данных (правило смешения, и его вариации, теория обобщенной проводимости и др. [1-4]), либо на основе физических моделей (закон Фурье, принцип локального термодинамического равновесия, система уравнений Максвелла - Каттанео и др. [5-7]). Однако при решении ряда задач, например, нестационарной теплопроводности и тепловой устойчивости возникают проблемы, приводящих к существенному отличию теории от экспериментально наблюдаемых результатов. Возникает ряд вопросов при расчете многослойных и композиционных материалов. В современной классической механике считается, что материальная точка имеет внутреннюю структуру [8], за счет чего обладает дополнительными степенями свободы. По аналогии с материальной точкой будем считать, что тепловой поток также имеет структуру. В работе получена система уравнений, получено решение в частном случае для системы, имеющей два разных механизма передачи теплоты, в стационарном случае. Показано, что полученная система может быть сведена к обобщенному уравнению Фурье, уравнению Фурье в стационарном случае и системе уравнений Максвелла - Каттанео. Рассмотрено два частных случая: неравновесная задача и стационарная задача. В первом случае введено понятие неравновесной температуры. Получено уравнение теплопроводности с источниковыми членами, которое говорит о том, что сначала тепловое равновесие устанавливается в каждом канале, а затем наступает и между каналами. Во втором случае учет многоканальности подтверждает волновой характер процесса: даже в одномерном стационарном случае получаем отличное от линейности решение ввиду свойств уравнений четвертого порядка. Ключевые слова: многоканальной модель теплопроводности, уравнение Максвелла Каттанео, уравнение Фурье, структура теплового потока, неравновесная температура, источниковые члены, уравнение теплопроводности.
\end{abstract}

\section{ВВЕДЕНИЕ}

При описании процессов теплопереноса используют разные модели, но все они имеют некоторые замечания и комментарии. Зачастую экспериментальные результаты описываются и эмпирическими моделями: правилом смешения, моделями теории обобщенной проводимости [1-4]. Однако, эти модели несовершенны и порой выбор конкретной модели аргументировать, кроме как согласи-

Степашкина Анна Сергеевна e-mail: stepashkina.anna@yandex.ru ем эксперимента и результатов моделирования, затруднительно.

В стационарном случае на макроскопическом уровне теплоперенос часто удается описать с помощью закона Фурье, согласно которому плотность тепла потока пропорциональна градиенту температуры

$$
q=-\lambda \cdot \operatorname{grad}(T),
$$

где $q-$ тепловой поток, $\lambda-$ коэффициент теплопроводности, $T$ - температура. Закон Фурье имеет ряд существенных недостатков. Описать распространение тепла в одномерном кристалле затруднительно, что не позволяет описывать процессы теплопереноса и

(i) Контент доступен под лицензией Creative Commons Attribution 4.0 License.

The content is available under Creative Commons Attribution 4.0 License. 


\section{A. C. Степашкина}

теплообмена на микроскопическом уровне. С развитием лазерных технологий появляется необходимость описывать практически мгновенное распространение теплоты на малых временных интервалах: расчетные данные в соответствии с законом Фурье существенно отличаются от экспериментальных.

Существуют и другие подходы, позволяющие описывать быстро протекающие тепловые процессы такие, как тепловой удар. Так В. А. Фок [9] предложил гиперболическое уравнение для передачи энергии, которое в последствии получило название волновое уравнение Клейна-Гордона - Фока. Используется для описания механики быстро движущихся частиц. Каттанео [10] эмпирически с учетом запаздывания была получена система уравнений

$$
\begin{gathered}
\tau_{p} \frac{\partial q}{\partial t}+q=-\lambda \operatorname{grad} T \\
\tau_{p} \frac{\partial^{2} T}{\partial t^{2}}+\frac{\partial T}{\partial t}=\operatorname{div}\left(a_{T} \cdot \operatorname{grad} T\right)
\end{gathered}
$$

где $a_{T}=\frac{\lambda}{c_{v} \rho}-$ коэффициент диффузии тепла (температуропроводности), $c_{v}$ - удельная теплоемкость, $\rho-$ плотность материала, $\tau_{p}$ время релаксации (затухание тепловой волны). Уравнение Каттанео есть обобщение закона Фурье с учетом того, что тепло распространяется с некоторой конечной скоростью. Система уравнений (1) позволяет описывать реальные эксперименты, в которых температура устанавливается в течение конечного времени релаксации.

Существуют методы, в которых процесс переноса тепла рассматривается локально-неравновесным [11-13]. Математические модели, описывающие локально-неравновесные процессы переноса, представляют собой иерархическую последовательность, соответствующую степени отклонения системы от локального равновесия. Степень отклонения от локального равновесия и адекватный выбор той или иной модели переноса зависят от соотношения между характерными пространственно-временными масштабами процесса переноса (макромасштабами) и микромасштабами, характеризующими релаксацию системы к локальному равновесию. Характерные масштабы процесса переноса зависят как от внешних факторов (граничные и начальные условия, источники тепла и массы), так и от параметров самой системы (коэффициенты диффузии, теплопроводность и т. д.).

Сложность решения задачи переноса теплоты в рамках моделей локального равновесия в корректном выборе времен релаксации и масштабов пространственной нелояльности к внутренним параметрам системы.

\section{1. МОДЕЛЬ МНОГОКАНАЛЬНОЙ ТЕПЛОПРОВОДНОСТИ}

\section{1. Основные положения многоканальной теплопроводности}

При описании процессов теплопереноса в твердых телах принято считать, что основным механизмом передачи тепловой энергии являются колебания кристаллической решетки, но вклад в это процесс вносят и электроны, в диэлектриках он пренебрежимо мал.

Практическим примером, подтверждающим экспериментально наличие у потока тепла структуры, являются полимерные композиционные материалы, имеющие гетерогенное строение. При исследовании теплофизических свойств композиций на основе полиолефиновой матрицы и современных углеродных наполнителей (графен, углеродные нановолокна и т. п.), обладающих высоким коэффициентом теплопроводности, установлено, что значение коэффициента теплопроводности значительно отличается от ожидаемого. Теплопроводность полиолефинов достигает 0.1-0.3 Вт/(м·К) [14], а углеродных нановолокон, например, $1200 \mathrm{Bт} /(\mathrm{M} \cdot \mathrm{K})$; значение теплопроводности композитного материала из этих веществ принимает значение 2-8 Вт/(м·К) [15], что достаточно сложно предсказать с помощью существующих физических моделей и теорий.

Можно говорить о том, что в веществах существует много «ящиков» (резервуаров) энергии в соответствии со статистикой Больцмана. Каждый такой ящик обладает своими теплофизическими свойствами, например, различными дисперсионными зависимостями. 
В соответствии с такими рассуждения в работах [11-13] предложена многотемпературная модель. В настоящей работе авторами также рассматривается решение задачи теплопроводности, однако вводится поправка на неравновесные процессы.

В классической термодинамике температура определяется только для равновесных процессов. В теплообменных процессах разные уровни «ящиков» энергии опустошаются по-разному.

Процесс теплопереноса будем рассматривать с позиции многоканального переноса [14]. Пусть тепловой поток распространяется по $N$ различным механизмам, которые и будут называться каналами распространения теплоты. Каждый канал характеризуется определенным физическим процессом, механизмом переноса, скоростью распространения тепла. Количество теплоты на каждом канале определяет структуру теплового потока.

\section{2. Уравнение теплопроводности в многоканальной модели}

Рассмотрим самый простой случай: изолированную систему с $N$ механизмами передачи теплоты на примере одномерной задачи. Система изолированная, поэтому теплообмен с окружающей средой отсутствует. Поток тепла в каждом канале праспространяется вдоль оси $O X$ как по ее направлению, так и против. Введем плотности потоков тепла $q_{\vec{n}}$ и $q_{\bar{n}}$, распространяющихся по $q_{\vec{n}}$ и против оси $q_{\bar{n}}$ заданной оси по $N$ каналам распространения (всего $2 N$-величин) в двухпотоковом приближении.

Запишем общий вид плотности теплового потока в системе:

$$
q(x, t)=\sum_{n=1}^{N} q_{\bar{n}}(x, t)-q_{\bar{n}}(x, t) .
$$

Переносимая часть внутренней энергии $U(x, t)$ положим равной сумме энергий на каждом канале:

$$
\begin{gathered}
U(x, t)=\sum_{n=1}^{N}\left[u_{\vec{n}}(x, t)+u_{\bar{n}}(x, t)\right]= \\
=\sum_{n=1}^{N} \frac{1}{c_{n}}\left[q_{\vec{n}}(x, t)+q_{\bar{n}}(x, t)\right],
\end{gathered}
$$

где $c_{n}$ - скорость распространения тепла в $n$-м канале. Таким образом, можно говорит о том, что каждый канал обладает собственной частотой, соответственно имеет место понятие локальной температуры.

Передача теплоты - процесс необратимый и неравновесный. При нестационарном теплопереносе имеют место два физических процесса: первый - переда энергии от более нагретого участка к менее нагретому, второй - обмен энергией между различными каналами распространения.

Для вывода уравнений теплопереноса в общем случае достаточно записать баланс энергий, который представляет собой систему из $2 N$ уравнений.

$$
\begin{aligned}
\frac{d u_{\vec{n}}}{d t}= & -\frac{d q_{\vec{n}}}{d x}-\sum_{m=1}^{N}\left[a_{n \bar{m}}+b_{n \bar{m}}\right] q_{\vec{n}}+ \\
& +\sum_{m=1}^{N} b_{n \bar{m}} q_{\bar{m}}-\sum_{m=1}^{N} a_{n \bar{m}} q_{\bar{m}} \\
\frac{d u_{\bar{n}}}{d t}= & -\frac{d q_{\bar{n}}}{d x}-\sum_{m=1}^{N}\left[a_{n \bar{m}}+b_{n \bar{m}}\right] q_{\bar{n}}+ \\
& +\sum_{m=1}^{N} b_{n \bar{m}} q_{\bar{m}}-\sum_{m=1}^{N} a_{n \bar{m}} q_{\bar{m}} .
\end{aligned}
$$

Слагаемое $\frac{d q_{\vec{n}}}{d x}$ отвечает за поглощенную долю энергии потоком, $a_{n m}$ - доля тепловой энергии, перешедшей с одного потока на другой с изменением направления распространения, $b_{n m}$ - доля энергии, перешедшей с одного потока на другой без изменения направления движения, причем $b_{n n}=0$.

Уравнения системы выражают перенос энергии с учетом внутреннего обмена между каналами. Введем следующие параметры: матрицу рассеяния

$$
\Theta_{k n}=c_{k} \sum_{m=1}^{N}\left\{\left[a_{k m}+b_{k m}\right] \delta_{k n}+\left(a_{k n}-b_{k n}\right)\right\} .
$$

и матрицу передачи энергии

$$
G_{k n}=c_{k} \sum_{m=1}^{N}\left\{\left[a_{k m}+b_{k m}\right] \delta_{k n}-\left(a_{k n}+b_{k n}\right)\right\} .
$$

Здесь $\delta_{k n}-$ дельта-функция, $c_{k}-$ скорость распространения теплоты в канале.

В полном равновесии в соответствии с принципом детального равновесия сумма энергии, пришедшей на канал $N$ равна той 


\section{A. C. Степашкина}

энергии, которая ушла с канала $(N-1)$. Таким образом матрицы $\Theta_{k n}$ и $G_{k n}$ являются симметричными.

Путем алгебраических преобразований система уравнений (2) принимает вид:

$$
\begin{gathered}
\frac{d u_{n}}{d t}+\frac{d q_{n}}{d x}+\sum_{k=1}^{N} u_{k} G_{k n}=0 \\
\frac{d q_{n}}{d t}+c_{n}^{2} \frac{d u_{n}}{d x}+\sum_{k=1}^{N} q_{k} \Theta_{k n}=0 .
\end{gathered}
$$

Первая группа $N$ уравнений, полученная путем сложения уравнений системы (3), учитывает внутренние переходы энергии с одного канала на другой, таким образом, мы получаем уравнение непрерывности, закон сохранения энергии.

Вторая группа уравнений, полученная в результате разницы уравнений системы (3), выражают обобщенный закон Фурье. Система уравнений (3) сводится к системе уравнений Каттанео (1) [15].

В стационарном случае, когда между каналами установилось равновесие, уравнения вторая группа уравнений системы (3) сводится к закону Фурье:

$$
q_{n}=-\Theta_{k n}^{-1} c_{n} \frac{d u_{n}}{d x} .
$$

Получим их системы уравнений (3) обобщенное уравнение Клейна - Гордона-Фока [16-18]. Для этого преобразуем: возьмем производную по времени от первого уравнения системы (3) и дивергенцию - от второго:

$$
\begin{gathered}
\ddot{u}_{n}+\nabla \dot{q}_{n}+\sum_{k=1}^{N} \dot{u}_{k} G_{k n}=0 \\
\nabla \dot{q}_{n}+c_{n}^{2} \Delta u_{n}+\sum_{k=1}^{N} \Theta_{k n} \cdot \nabla q_{k}=0 .
\end{gathered}
$$

Учитывая, что $\nabla q_{k}=\dot{u}_{k}-\sum_{k=1}^{N} u_{k} G_{k n}$ из первого уравнения системы (3)), найдем разность первого и второго уравнений системы (5):

$$
\begin{gathered}
\ddot{u}_{n}+\sum_{k=1}^{N} \dot{u}_{k} G_{k n}-c_{n}^{2} \Delta u_{n}-\sum_{k=1}^{N} \dot{u}_{k} G_{k n}- \\
-\sum_{k=1}^{N} \Theta_{k n} \cdot \sum_{k=1}^{N} u_{k} G_{k n}=0 .
\end{gathered}
$$

Примем $L_{k n}=\Theta_{k n} \cdot G_{k n}-$ матрица диссипации энергии. Выражение (5) принимает вид:

$$
\begin{gathered}
\ddot{u}_{n}+\sum_{k=1}^{N} \dot{u}_{k}\left(G_{k n}-\Theta_{k n}\right)-c_{n}^{2} \Delta u_{n}- \\
-\sum_{k=1}^{N} L_{k n} \cdot u_{k}=0 .
\end{gathered}
$$

Полученное уравнение (6) позволяет описать механическое движение тепловых потоков на каждом канале.

\section{2. РЕЗУЛЬТАТЫ И ИХ ОБСУЖДЕНИЕ}

\section{1. Уравнение многоканальной проводимости в случае двухканальной системы в неравновесном состоянии}

Ограничимся рассмотрением одномерной двухканальной системой для совершенных тел. Под совершенным телом понимается тело с постоянной теплоемкостью по температуре.

Температура определяет заселенность «ящиков» энергии в рассматриваемой системе. Введем неравновесную температуру путем введения неравновесной поправки к температуре. Общую энергию на каждом канале можно записать в виде

$$
u=C(T+\delta T)
$$

где $T-$ равновесная температура, $\delta T-$ неравновесная температура, $C=\frac{d u}{d t}-$ теплоемкость.

Существует два резервуара энергии с разными скоростями, например, решеточная и электронная теплопроводность, продольная и поперечная моды колебания в твердом теле.

Из системы уравнений (3) получаем

$$
\begin{aligned}
& \frac{d u_{1}}{d t}+\frac{d q_{1}}{d x}+c_{1} u_{1}\left(a_{12}+b_{12}\right)= \\
& \quad=c_{2} u_{2}\left(a_{21}+b_{21}\right) \\
& \frac{1}{c_{1}} \frac{\partial q_{1}}{\partial t}+\frac{d}{d x} c_{1} u_{1}+q_{1}\left(2 a_{11}+a_{12}+b_{12}\right)= \\
& \quad=q_{2}\left(b_{21}-a_{21}\right) \\
& \frac{d u_{2}}{d t}+\frac{d q_{2}}{d x}+c_{2} u_{2}\left(a_{21}+b_{21}\right)= \\
& =c_{1} u_{1}\left(a_{12}+b_{12}\right) \\
& \frac{1}{c_{2}} \frac{\partial q_{2}}{\partial t}+\frac{d}{d x} c_{2} u_{2}+q_{2}\left(2 a_{22}+a_{21}+b_{21}\right)= \\
& =q_{1}\left(b_{12}-a_{12}\right) .
\end{aligned}
$$


Первое и третье уравнения системы описывают переходы энергии с одного канала на другой с изменением или сохранением направления распространения теплового потока. Второе и четвертое уравнения учитывают переходы внутри потока.

В случае термодинамического равновесия системы согласно принципу детального равновесия, т. е. количество ушедшей энергии с первого канала на второй равно количеству пришедшей со второго канала на первый. В силу симметрии положим $b_{12}=a_{12}, b_{21}=a_{21}$. Система уравнений (7) принимает вид

$$
\begin{aligned}
& \frac{d u_{1}}{d t}+\frac{d q_{1}}{d x}+2 a_{12} c_{1} u_{1}=2 a_{21} c_{2} u_{2} \\
& \frac{1}{c_{1}} \frac{\partial q_{1}}{\partial t}+\frac{d}{d x} c_{1} u_{1}+q_{1}\left(2 a_{11}+2 a_{12}\right)=0 \\
& \frac{d u_{2}}{d t}+\frac{d q_{2}}{d x}+2 a_{21} c_{2} u_{2}=2 a_{12} c_{1} u_{1} \\
& \frac{1}{c_{2}} \frac{\partial q_{2}}{\partial t}+\frac{d}{d x} c_{2} u_{2}+q_{2}\left(2 a_{22}+2 a_{21}\right)=0 .
\end{aligned}
$$

Полученная система уравнений (8) есть не что иное, как классический закон теплопроводности Фурье. Из уравнений 2 и 4 системы (8) выразим значение потоков на каждом канале:

$$
\begin{aligned}
q_{1}= & -\frac{1}{2 a_{11}+2 a_{12}} \frac{\partial}{\partial x} c_{1} u_{1}- \\
& -\frac{1}{c_{2}\left(2 a_{22}+2 a_{21}\right)} \frac{\partial q_{1}}{\partial t} \\
q_{2}= & -\frac{1}{2 a_{22}+2 a_{21}} \frac{\partial}{\partial x} c_{2} u_{2}- \\
& -\frac{1}{c_{2}\left(2 a_{22}+2 a_{21}\right)} \frac{\partial q_{2}}{\partial t}
\end{aligned}
$$

Введем переобозначения:

1) коэффициент температуропроводности в каналах

$$
A_{1}=\frac{c_{1}}{2 a_{11}+2 a_{12}}, A_{2}=\frac{c_{2}}{2 a_{22}+2 a_{21}} .
$$

2) времена релаксации на каждом канале:

$$
\begin{gathered}
\tau_{1}=-\frac{1}{c_{1}\left(2 a_{11}+2 a_{12}\right)} \frac{d q_{1}}{d t}, \\
\tau_{2}=-\frac{1}{c_{2}\left(2 a_{22}+2 a_{21}\right)} \frac{d q_{2}}{d t} .
\end{gathered}
$$

На больших временных интервалах релаксационный параметр считаем малым [19]. Поскольку мы рассматриваем систему на макроуровне, то параметрами $\tau_{1}, \tau_{2}$ пренебрегаем. Их влияние существенно на малом масштабе: задача в масштабе кристаллической решетке, когда масштабы перехода с одного канала на другой сопоставимы с длиной свободного пробега. С учетом вышеизложенного система уравнений (9) принимает вид:

$$
\begin{aligned}
& q_{1}=-A_{1} \frac{d u_{1}}{d x} \\
& q_{2}=-A_{2} \frac{d u_{2}}{d x}
\end{aligned}
$$

Рассмотрим 1 и 3 уравнения системы уравнений (8). Учитывая выражения для потока (10) получаем

$$
\begin{aligned}
& \frac{d u_{1}}{d t}-A_{1} \frac{d^{2} u_{1}}{d x^{2}}+2 a_{12} c_{1} u_{1}=2 a_{21} c_{2} u_{2} \\
& \frac{d u_{2}}{d t}-A_{2} \frac{d^{2} u_{2}}{d x^{2}}+2 a_{21} c_{2} u_{2}=2 a_{12} c_{1} u_{1} .
\end{aligned}
$$

Полная энергия системы $U=u_{1}+u_{2}$. Сложим уравнения системы (11):

$$
\frac{d U}{d t}=A_{1} \Delta u_{1}+A_{2} \Delta u_{2} .
$$

Для равновесной температуры в абсолютно твердом теле энергия связана с равновесным значением температуры $T_{p}$ выражением

$$
U=C T_{p} .
$$

Введем обобщенный коэффициент температуропроводности

$$
A=\frac{C_{1} A_{1}+C_{2} A_{2}}{C_{1}+C_{2}} .
$$

Учитывая вышеизложенное, уравнение (12) принимает вид

$$
\frac{d T_{p}}{d t}=A \Delta T_{p} .
$$

Получили классическое уравнение Фурье. В реальном мире равновесных процессов не существует, поэтому рассмотрим температуру как параметр, имеющий равновесную $T_{p}$ и неравновесную составляющие $\delta T$. Внутренняя энергия для каждого канала может быть представлена в виде:

$$
u_{i}=C_{i}\left(T_{p}+\delta T\right) .
$$




\section{А. С. Степашкина}

Произведем следующее преобразование системы уравнений (9): вычтем из третьего уравнения системы первое. Получаем

$$
\begin{gathered}
\left(C_{1}-C_{2}\right) \frac{d}{d t}\left(T_{p}+\delta T\right)- \\
-\left(C_{1} A_{1}-C_{2} A_{2}\right) \Delta\left(T_{p}+\delta T\right)= \\
=4\left(T_{p}+\delta T\right)\left(C_{2} a_{21} c_{2}-C_{1} a_{12} c_{1}\right) .
\end{gathered}
$$

Введем параметры

1) нестационарную температуропроводность

$$
\delta A=\frac{C_{1} A_{1}+C_{2} A_{2}}{C_{1}+C_{2}},
$$

2) частоту переходов между каналами

$$
B=\frac{C_{2} a_{21} c_{2}-C_{1} a_{12} c_{1}}{C_{2}-C_{1}} .
$$

Система уравнений (9) с учетом преобразований может быть представлена в виде двух уравнений, позволяющий описать процесс теплопроводности для равновесного и неравновесного случая

$$
\begin{aligned}
& \frac{d T_{p}}{d t}=A \Delta T_{p} \\
& \frac{d}{d t}\left(T_{p}+\delta T\right)=\delta A \Delta\left(T_{p}+\delta T\right)+B\left(T_{p}+\delta T\right) .
\end{aligned}
$$

Подставим первое уравнение системы (13) во второе, получим уравнение теплопроводности с источниковыми членами

$$
\frac{d}{d t} \delta T-\delta A \Delta \delta T+B \delta T=A \Delta T_{p}-B T_{p} .
$$

Выражение (14) говорит о том, что сначала тепловое равновесие устанавливается в каждом канале, а затем наступает и между каналами. Примером является металл, в кристаллической решетке сначала устанавливается тепловое равновесие электронного газа, а затем всей системы в целом [20-22].

\section{2. Решение задачи многоканальной теплопроводности для одномерного стационарного случая}

В качестве примера рассмотрим передачу теплового потока в металлическом стержне, ограниченного диэлектриками (металлоидами). Металлический стержень - это одномерный объект длиной $a$ с двумя явно выра- женными резервуарами энергии, связанными с решеткой и свободными электронами. Диэлектрики, с помощью которых поддерживается температура на концах стержня обладают только решеточным типом проводимости. Внутренняя энергия в двухканальной системе определяется теплопроводностью в каналах и обменом энергии между каналами.

Рассмотрим решение системы уравнений (10), (11):

$$
\begin{gathered}
q_{e}=-A_{e} \frac{d u_{e}}{d x} \\
q_{h}=-A_{h} \frac{d u_{h}}{d x} \\
\frac{d u_{e}}{d t}-A_{e} \frac{d^{2} u_{e}}{d x^{2}}+2 a_{12} c_{e} u_{e}=2 a_{21} c_{h} u_{h} \\
\frac{d u_{h}}{d t}-A_{h} \frac{d^{2} u_{h}}{d x^{2}}+2 a_{21} c_{h} u_{h}=2 a_{12} c_{e} u_{e} .
\end{gathered}
$$

Индекс е обозначает параметры канала, где механизм передачи теплоты - электронный, а $h$ - для решеточного механизма.

Запишем граничные условия с учетом стационарности задачи:

$$
\begin{aligned}
& \left.T\right|_{x=0}=T_{1}(t)=T_{1}=\mathrm{const} \\
& \left.T\right|_{x=a}=T_{2}(t)=T_{2}=\mathrm{const} .
\end{aligned}
$$

Поскольку мы рассматриваем решение стационарной задачи, то можно говорить об установившемся суммарном тепловом потоке.

Введем коэффициента обмена энергией между каналами

$$
\begin{aligned}
& K_{e}=2 a_{12} c_{e} \\
& K_{h}=2 a_{21} c_{h} .
\end{aligned}
$$

Тогда система уравнений (15) принимает вид

$$
\begin{gathered}
q_{e}=-A_{e} \frac{d u_{e}}{d x} \\
q_{h}=-A_{h} \frac{d u_{h}}{d x} \\
-A_{e} \frac{d^{2} u_{e}}{d x^{2}}+K_{e} u_{e}=K_{h} u_{h} \\
-A_{h} \frac{d^{2} u_{h}}{d x^{2}}+K_{h} u_{h}=K_{e} u_{e} .
\end{gathered}
$$

Учитывая, что $u_{i}=C_{i} T_{i}, \lambda_{i}=A_{i}$ система (16) может быть переписана следующим образом 


$$
\begin{gathered}
q_{e}=-\lambda_{e} \frac{d u_{e}}{d x} \\
q_{h}=-\lambda_{h} \frac{d u_{h}}{d x} \\
-C_{e} A_{e} \frac{d^{2} T_{e}}{d x^{2}}+K_{e} C_{e} T_{e}=K_{h} C_{h} T_{h} \\
-C_{h} A_{h} \frac{d^{2} T_{h}}{d x^{2}}+K_{h} C_{h} T_{h}=K_{e} C_{e} T_{e} .
\end{gathered}
$$

Введем обозначения

$$
\begin{aligned}
& \alpha_{e}=\frac{K_{e}}{A_{e}}, \beta_{e}=\frac{K_{e} C_{e}}{A_{h} C_{h}}, \\
& \alpha_{h}=\frac{K_{h}}{A_{h}}, \beta_{h}=\frac{K_{h} C_{h}}{A_{e} C_{e}} .
\end{aligned}
$$

Система уравнений (17) принимает вид

$$
\begin{gathered}
q_{e}=-\lambda_{e} \frac{d u_{e}}{d x} \\
q_{h}=-\lambda_{h} \frac{d u_{h}}{d x} \\
-\frac{d^{2} T_{e}}{d x^{2}}+\alpha_{e} T_{e}=\beta_{h} T_{h} \\
-\frac{d^{2} T_{h}}{d x^{2}}+\alpha_{h} T_{h}=\beta_{e} T_{e} .
\end{gathered}
$$

Преобразуем третье и четвертое уравнения системы, путем подстановки четвертого уравнения в третье, получаем

$$
\frac{d^{4} T_{h}}{d x^{4}}-\left(\alpha_{e}+\alpha_{h}\right) \frac{d^{2} T_{h}}{d x^{2}}+\left(\alpha_{e} \alpha_{h}-\beta_{e} \beta_{h}\right) T_{h}=0 .
$$

Введем переобозначения:

$$
\begin{gathered}
\vartheta=-\left(\alpha_{e}+\alpha_{h}\right), \\
\tau=\alpha_{e} \alpha_{h}-\beta_{e} \beta_{h}, \\
\mu=\frac{d T_{h}}{d x}, \omega=\frac{d^{2} T_{h}}{d x^{2}} .
\end{gathered}
$$

и решим биквадратное уравнение

$$
\omega^{2}+\vartheta \omega+\tau=0
$$

Найдем дискриминант уравнения

$\Delta=\vartheta^{2}-4 \tau=\left(\alpha_{e}+\alpha_{h}\right)^{2}-4\left(\alpha_{e} \alpha_{h}-\beta_{e} \beta_{h}\right)$.

Решение уравнения может быть записано в виде

$$
\begin{aligned}
& \omega_{12}=\frac{1}{2}\left[-\vartheta \pm \sqrt{\vartheta^{2}-4 \tau}\right] \\
& \mu_{12}= \pm \sqrt{\frac{1}{2}[-\vartheta+\sqrt{\Delta}]} i \\
& \mu_{34}= \pm \sqrt{\frac{1}{2}[-\vartheta-\sqrt{\Delta}]} i
\end{aligned}
$$

Решение уравнения является следующее выражение для $T_{h}$

$T_{h}=B_{1} \cos \left(\mu_{12} x+\phi_{1}\right)+B_{2} \cos \left(\mu_{34} x+\phi_{2}\right)$.

$\mathrm{B}$ соответствии с решением уравнения теплопроводности можно говорит о том, что на каждом канале температура изменяется нелинейно. Получаем области с большей температурой на одном канале.

Реальные эксперименты показывают, что в линейной системе при установившемся стационарном режиме, температура меняется нелинейно [20-22]. Учет многоканальности подтверждает волновой характер процесса: даже в одномерном стационарном случае получаем отличное от линейности решение ввиду свойств уравнений четвертого порядка.

\section{ЗАКЛЮЧЕНИЕ}

В работе предложен метод описания процесса теплопереноса с точки зрения многоканальной модели, в соответствии с которой распространение теплоты осуществляется по двум разным механизмам передачи, причем каждому механизму соответствует свой канал. Показано, что полученная система может быть сведена к обобщенному уравнению Фурье, уравнению Фурье в стационарном случае и системе уравнений Каттанео. Рассмотрено два частных случая: неравновесная задача, стационарная задача.

Рассмотрено два частных случая: неравновесная задача, стационарная задача на примере двухкомпонентной (двухканальной) модели. Введена неравновесную температуру состоящую из суммы температуры и неравновесной поправки к ней. В таком случае, было получено уравнение теплопроводности с источниковыми членами.

В соответствии с решением уравнения теплопроводности многоканальной теплопроводности в стационарном случае можно говорить о том, что в каналах температура изменяется нелинейно.

\section{КОНФЛИКТ ИНТЕРЕСОВ}

Автор декларирует отсутствие явных и потенциальных конфликтов интересов, связанных с публикацией настоящей статьи. 


\section{А. C. Степашкина}

\section{СПИСОК ЛИТЕРАТУРЫ}

1. Lichtenecker, K. The Electrical Conductivity of Periodic and Random Aggregates / K. Lichtenecker // PhysikalischeZeitschrift. - 1924. Vol. 25. - P. 169.

2. Митюшов, E. А. Обобщенная проводимость и упругость микронеоднородных гетерогенных материалов / Е. А. Митюшов, П. В. Гельд, Р. А. Адамеску. - М. : Металлургия, 1998. - 143 с.

3. Оделевский, В. И. Расчет обобщенной проводимости гетерогенных систем / В. И. Оделевский // Журнал Технической Физики. - 1951. - Т. 21, вып.6. - С. 667-685.

4. Girifalco L. A. Statistical Physics of materials / L. A Girifalko. - New York : Wiley, 1973.

5. Вейник, А. И. Термодинамика. 3-е изд. / А. И. Вейник - Минск : Вышейшая школа, 1968. - 464 c.

6. Босворт, Р. Процессы теплового переноса / Р. Босворт. - Москва : Гостехиздат, 1957. - $876 \mathrm{c}$.

7. Льков, А. В. Теория теплопроводности / А. В. Лыков. - Москва : Высшая школа, 1967. $600 \mathrm{c}$.

8. Пальмов, В. А. Фундаментальные законы природы в нелинейной термомеханике деформируемых тел: Учебное пособие / В. А. Пальмов. - СПб. : Изд-в СПбГПУ, 2008. $143 \mathrm{c}$.

9. Фок, В. А. Решение одной задачи теории диффузии по методу конечных разностей и приложение его к диффузии света / В. А. Фок // Труды ГОИ. - 1926. - Т. 4, вып. 34. - С. 1-32.

10. Cattaneo, C. AttiSeminarioUniv. Modena. 1948. - Vol. 33. - P. 3.

11. Соболев, С. Л. Локально-неравновесные модели процессов переноса / С. Л. Соболев // Успехи физических наук. - 1997. - Т. 167, № 10. - C. 1095-1106.

12. Sobolev, S. L. Nonlocal diffusion models: Application to rapid solidification of binary mixtures / S. L. Sobolev International Journal of Heat and Mass Transfer. - 2014. - V. 71. - P. 295-302. DOI: 10.1016/j.ijheatmasstransfer.2013.12.048

13. Sobolev, S. L. Two-temperature model for nonlocal heat conduction / S. L. Sobolev // Journal of Physics III. - 1993. - V. 3. - P. 2261-2269.
14. Stepashkina, A. S. Heat distribution with structure in solid states / A. S. Stepashkina, P. P Rymkevich, A. V. Kotskovich, A. I. Altukhov // Material physics and mechanics. - 2017. V. 31. - P. 75-77.

15. Tsobkallo, E. S. Transenergo Plastic based on Film-Type Composite Materials / E. S. Tsobkallo, O. A. Moskalyuk, A. S. Stepashkina, V. Ye. Yudin // Fiber Chemistry. - 2018. - V. 50, No 4. - P. 325-331. - DOI: 10.36807/1998-98492020-52-78-28-35

16. Fock, V. Zur Schrödingerschen Wellenmechanik / Zeitschrift für Physik // V. Fock. 1926. - V. 38. - P. 242-250.

17. Klein, O. Quantentheorie und fünfdimensionale Relativitätstheorie / O. Klein // Zeitschrif tfür Physik. - 1926. - V. 37. - P. 895-906.

18. Gordon W. Der Comptoneffektnach der Schrödingerschen Theorie / W. Gordon // Zeitschrift für Physik. 1926. - V. 40, iss. 1. P. 117-133.

19. Тихонов, А. Н. Уравнения математической физики / А. Н. Тихонов, А. А. Самарский. - М. : Наука, 1972. - 736 с.

20. Военков, Н. В. Экспериментальное моделирование и теоретический анализ термодеформации пластин диэлектрических материалов при субмикросекундных длительностях радиационного нагрева / Н. В. Военков, Б. А. Зимин, Ю. В. Судьенков // Журнал технической физики. - 2011. - т. 81, вып. 8. C. $69-75$. DOI: $10.1134 / \mathrm{S} 1063784211070255$

21. Военков, Н. В. Экспериментальные исследования термоупругих напряжений в тепло- и нетеплопроводящих твердых телах при субмикросекундных длительностях лазерного нагрева / Н. В. Военков, Б. А. Зимин, Ю. В. Судьенков // Журнал технической физики. - 2011. - т. 81, вып. 6. - С. 57-62. DOI: $10.1134 /$ S1063784211060296

22. Военков, Н. В. Особенности формирования динамических напряжений в тепло- и нетеплопроводящих материалах при субмикросекундных длительностях нагрева / Н. В. Военков, Б. А. Зимин, Ю. В. Судьенков // Вестник СПбГУ. - 2008. - Сер.1, вып. 4. C. $110-117$. 
Степашкина Анна Сергеевна - канд. техн. наук, доцент кафедры № 6 метрологического обеспечения инновационных технологий и промышленной безопасности института фундаментальной подготовки и технологических инноваций Санкт-Петербургского государственного университета аэрокосмического приборостроения.

E-mail: stepashkina.anna@yandex.ru

ORCID iD: https://orcid.org/0000-0003-3326-0776

DOI: https://doi.org/10.17308/sait.2021.3/3732

Received 05.04.2021

Accepted 20.11.2021

\title{
MULTICHANNEL MODEL FOR THERMAL CONDUCTIVITY PROCESS
}

\author{
(c) 2021 A. S. Stepashkina ${ }^{\bowtie}$ \\ State University of Aerospace Instrumentation \\ 67, Bolshaya Morskaya Street, 190000 Saint Petersburg, Russian Federation
}

\begin{abstract}
Annotation. A description of the heat transfer process at the macroscopic level occurs using wellknown classical methods and theories found by approximating experimental data (the mixing model and its variations, theory of generalized conductivity, etc.[1-4]) or on the basis of physical models (Fourier's Law, the principle of the local thermodynamic equilibrium, the Maxwell Cattaneo system of equations, etc. [5-7]). When we solve a number of problems connected with unsteady thermal conductivity and thermal stability, we have a significant difference between the theory and the experimentally observed results. A number of questions arise when we calculate multilayer and composite materials' thermal properties. In modern classical mechanics it is believed that a material point has an internal structure [8] due to which it has additional degrees of freedom. We assume that the heat flux also has a structure by analogy with a material point. In this work a multichannel heat conduction equation (and its solution) is obtained in a particular case for a system with two different heat transfer mechanisms in the stationary case. It is shown that the resulting system can be reduced to the generalized Fourier equation, the Fourier equation in the stationary case and the Maxwell - Cattaneo system of equations. Two special cases are considered: a nonequilibrium problem and a stationary problem. In the first case the concept of non-equilibrium temperature is introduced. The equation of heat conduction with source terms is obtained. It allows to say that first thermal equilibrium is established in each channel and then it occurs between the channels. The second case shows that taking multichannel into account confirms the wave character of the process. Even in the one-dimensional stationary case we obtain a nonlinear solution of such fourth-order properties.

Keywords: multichannel heat conduction model, Maxwell - Cattaneo equation, Fourier Law, heat flow structure, nonequilibrium temperature, source terms, heat conduction equation.
\end{abstract}

\section{CONFLICT OF INTEREST}

The authors declare the absence of obvious and potential conflicts of interest related to the publication of this article.

Stepashkina Anna S.

e-mail: stepashkina.anna@yandex.ru

\section{REFERENCES}

1. Lichtenecker K. (1924) The Electrical Conductivity of Periodic and Random Aggregates. Physikalische Zeitschrift. (25). P. 169-179.

2. Mityushov Ye. A. et al. (1998) Generalized conductivity and elasticity of microinhomogeneous heterogeneous materials. Moscow, Metallurgiya. (in Russian)

3. Odelevsky V. I. (1951) Calculation of the generalized conductivity of heterogeneous sys- 


\section{А. C. Степашкина}

tems. Journal of Technical Physics. 21(6). P. 667685.

4. Girifalco L. A. (1973) Statistical Physics of materials. New York: Wiley.

5. Veynik A. I. (1968) Thermodynamics. Minsk, Visheyshaya shkola. (in Russian)

6. Bosworth R. (1957) Thermal transfer process. Moscow, Gostechizdat. (in Russia)

7. Likov A. V. (1967) Theory of thermal conductivity. Minsk, Visheyshaya shkola. (in Russian)

8. Palmov V. A. (2008) Fundamental laws of nature in nonlinear thermomechanics of deformable bodies. SPb: SPbSPU Publishing House. (in Russian)

9. Fock V. A. (1926) Solution of one problem of the theory of diffusion by the method of finite differences and its application to diffusion of light. Proceedings of GOI. 4 (34). P. 1-32. (in Russian)

10. Cattaneo C. (1948) Atti Seminario Univ. Modena (33). P. 3-20.

11. Sobolev S. L. (1997) Locally nonequilibrium models of transfer processes. Advances in physical sciences. 167 (10). P. 1095-1106.

12. Sobolev S. L. (2014) Nonlocal diffusion models: Application to rapid solidification of binary mixtures. International Journal of Heat and Mass Transfer (71). P. 295-302. DOI:10.1016/j. ijheatmasstransfer.2013.12.048

13. Sobolev S. L. (1993) Two-temperature model for nonlocal heat conduction. Journal of Physics III (3). P. 2261-2269.

14. Stepashkina A. S. \& Rymkevich P. P. et al. (2017) Heat distribution with structure in solid states. Material physics and mechanics (31). P. 75-77.
15. Tsobkallo E. S \& Moskalyuk O. A. et al. (2018) Transenergo Plastic based on Film-Type Composite Materials. Fiber Chemistry. 50 (4). P. 325-331. DOI: 10.36807/1998-9849-2020-5278-28-35

16. Fock V. (1926) Zur Schrödingerschen Wellenmechanik. Zeitschrift für Physik. (38). P. 242-250.

17. Klein O. (1926) Quantentheorie und fünfdimensionale Relativitätstheorie. Zeitschrif tfür Physik (37). P. 895-906.

18. Gordon W. (1926) Der Comptoneffektnach der Schrödingerschen Theorie. Zeitschrift für Physik. 40 (1). P. 117-133.

19. Tikhonov A. N. \& Samara A. A. (1972) Equations of mathematical physics. Moscow : Nauka. (in Russian)

20. Vovnenko N. V., Zimin B. A. \& Sud'enkov Y. V. (2011) Experimental simulation and theoretical analysis of the thermal deformation of dielectric plates under submicrosecond radiation heating. Technical Physics. 56 (7). P. 968-974. DOI: $10.1134 /$ S1063784211070255

21. Vovnenko N. V., Zimin B. A. \& Sud'enkov Y. V. (2011) Experimental study of thermoelastic stresses in heat-conducting and non-heat-conducting solids under submicrosecond laser heating. Technical Physics. 56 (6). P. 803-808. DOI: 10.1134/S1063784211060296

22. Vovnenko N. V., Zimin B. A. \& Sud'enkov Y. V. (2008) Speciality of generation of dynamics stresses in heat-conducting and low heat-conducting media under sumicrosecond heating rate. Vestnik of the St.Petersburg university: mathematics 1(4). P. 110-117. (in Russian)

Stepashkina Anna S. - PhD, Associated Professor, Department №6 of Metrological Support of Innovation Technologies and Industrial Security, Institute of Fundamental Training and Technological Innovations, Saint Petersburg State University of Aerospace Instrumentation.

E-mail: stepashkina.anna@yandex.ru

ORCID iD: https://orcid.org/0000-0003-3326-0776 